\title{
KINEMATIC SYNTHESIS OF PLANAR ADJUSTABLE SIX BAR MECHANISM FOR SPECIFIED OUTPUT
}

\author{
C. M. Veerendrakumar ${ }^{1}$, V. B. Math $^{2}$ \\ ${ }^{1}$ Department of Automobile Engineering, Research Scholar,Basaveshwar Engineering College, Bagalkot-587102, \\ Karnataka, India \\ cmveerendra@gmail.com
}

\begin{abstract}
A method for the synthesis of planar adjustable six bar mechanism with a slider output is presented. The multi-loop six bar linkage considered here is a series combination of two four link mechanisms with first phase having regular RRRR linkage. The second phase consists of a slider crank with the slider being the output member. An analytical method is presented for the kinematic synthesis of a planar four bar adjustable mechanisms using Chebychev's accuracy points and Fruedenstein's equations for three point function generation that satisfy a given input output relationship. The length of the coupler link is taken as the adjustable parameter. An analytical method of computing the value of this variable for a four bar crank rocker mechanism as well as a slider crank mechanism is presented. A numerical example explains the synthesis procedure developed in which a function to be generated is considered. The unknown link lengths of the mechanism are then found for given range of input and output link parameters and assumed link length. The value of the adjustable length of the coupler is then computed and the results are presented numerically as well as graphically. The method adopted is coded using MATLAB for quick computation and ease of use. The motion and the path generated by the designed mechanism is found for different values of the adjusted parameter.
\end{abstract}

Keywords: Planar six mechanism, Adjustable link, Frueudenstein's method, Chebychev spacing, multi-loop linkage synthesis

\section{INTRODUCTION}

Machines have been used by humans since time immemorial to supplement the limited power they possess. The design of such machines and the mechanisms needed for them has been an integral part of the profession of mechanical engineering. With the recent trend of increase in the level of automation and mechanization in industries, the importance and scope for the design of mechanisms has increased tremendously. With the current demand on accuracy and speed of operation of modern machinery, a systematic approach towards their design has become more essential than ever (Kota S. and Soni A. H. 1992). Today the researchers are busy inventing innovative methods of design of machines employing more powerful computational facilities and easy to program analytical methods that are available to them. Computers are extensively employed now in the synthesis, analysis phases of design of machines as well as in their manufacturing and product life cycle maintenance. Kinematic analysis and synthesis play a fundamental role in the design of mechanisms for achieving an optimal solution.

Kinematicians world over have been fascinated by the idea of adjustable mechanisms and a number of researchers have worked on this concept. The ability of mechanisms to generate various kinds of motion can be greatly enhanced if they are made adjustable. Adjustable mechanisms also termed as reconfigurable or programmable mechanisms, are those in which one or more of their parameters are made adjustable. Majority of the initial work was on the graphical methods of synthesis but the availability of more powerful computational facilities paved the way for development of analytical techniques. With shorter life cycles becoming more common these days, the need for flexible manufacturing systems with adjustable linkage design has become more essential than ever.

Several researchers have tried to adjust a variety of parameters to synthesize adjustable mechanisms for function, path and motion generation. (Bonnell, R. D., and Cofer, J. A., 1966) pioneered the kinematic synthesis of adjustable four bar mechanisms by employing the complex number method developed by George N. Sandor. Later Joseph F. McGovern and George N. Sandor extended this work to geared linkages and higher order synthesis (Joseph F. McGovern and George N. Sandor, 1973). They developed a method of synthesizing adjustable planar linkages for function generation with finitely separated precision points and higher order synthesis involving prescribed velocities, accelerations, and higher accelerations. The method of synthesis is analytical with closed form solutions and utilizes complex numbers. The method is programmed for automatic digital computation.Terry E Shoup developed a technique for the design of an adjustable spatial slider crank mechanism to be used in applications such as variable displacement pump or compressor (Shoup Terry E., 1984). D. P. Naik and C. Amarnath applied theories of multidegree freedom systems to adjustable mechanisms and synthesized a four bar adjustable function generator 
operating in two phases to produce two specified functions which is based on five bar linkage theory (Naik D. P., Amarnath C., 1989).T. Chuenchom and S. Kota introduced the concept of Adjustable Robotic Mechanisms (ARMs) that are the true middle ground between conventional mechanism based hard automation and overly flexible anthropomorphic robots (Chuenchom T., Kota S., 1997).

The present work focuses on the design of planar adjustable six bar mechanism with a slider output. The multi loop linkage having two phases in which a regular RRRR linkage with the follower link becoming the common link with second phase. The second phase is having slider output. The length of the coupler is taken as the adjustable parameter.

\section{METHODOLOGY}

The synthesis procedure involves finding the dimensions of a mechanism capable of generating a desired function. The well established Freudenstein's equations are used to obtain the link lengths of planar adjustable mechanisms. Equations are then derived to find the variation of link length of the adjustable mechanism. The motion and path generated by this designed mechanism are determined. The developed procedure is then coded for quick computation using MATLAB.

Fig.1. shows a six bar planar adjustable mechanism with the slider output. It is composed of two loops in which the first loop $\mathrm{O}_{2} \mathrm{ABO}_{4}$ is a four bar mechanism with $\mathrm{O}_{2} \mathrm{~A}$ is the crank that makes an initial angle of $\theta_{2}$ with the fixed ground link $\mathrm{O}_{2} \mathrm{O}_{4}$. The coupler $\mathrm{AB}$ makes an angle $\theta_{4}$ with the ground link. The second phase of the linkage comprised of the loop $\mathrm{O}_{4} \mathrm{BCD}$ in which $\mathrm{O}_{4} \mathrm{~B}$ which is the follower of the first loop becomes the crank for the secondloop. Table-1 gives the details of the angles, link lengths, known and unknown values of the link parameters.

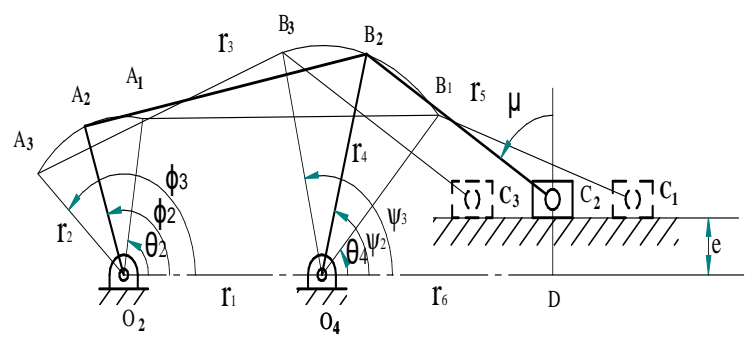

Fig.1. Six bar mechanisms with slider output

Table-1. Parameters of the planar adjustable six bar slider crank mechanism

\begin{tabular}{|l|l|l|l|l|l|}
\hline $\begin{array}{l}\text { Phas } \\
\text { e }\end{array}$ & Loop & $\begin{array}{l}\text { Inpu } \\
\mathbf{t} \\
\text { Angl } \\
\mathbf{e}\end{array}$ & $\begin{array}{l}\text { Outpu } \\
\mathbf{t} \\
\text { Angle }\end{array}$ & $\begin{array}{l}\text { Selecte } \\
\text { d Link } \\
\text { length }\end{array}$ & $\begin{array}{l}\text { Calculate } \\
\mathbf{d} \text { link } \\
\text { lengths }\end{array}$ \\
\hline \multirow{4}{*}{$\mathbf{I}$} & $O_{2} A_{1} B_{1} O_{4}$ & $\theta_{2}$ & $\theta_{4}$ & & \\
\cline { 2 - 4 } & $O_{2} A_{2} B_{2} O_{4}$ & $\phi_{2}$ & $\psi_{2}$ & $\mathrm{r}_{1}$ & $\mathrm{r}_{2}, \mathrm{r}_{3}$ and \\
$\mathrm{r}_{4}$
\end{tabular}

\begin{tabular}{|l|l|l|l|l|l|}
\hline & & & & & \\
\hline \multirow{4}{*}{ II } & $O_{4} B_{1} C_{1} D$ & $\theta_{4}$ & & & \\
\cline { 2 - 3 } & $O_{4} B_{2} C_{2} D$ & $\psi_{2}$ & $\begin{array}{l}\text { Slider } \\
\text { disp, S }\end{array}$ & $E$ & $\mathrm{r}_{5}$ \\
\cline { 2 - 3 } & $O_{4} B_{3} C_{3} D$ & $\psi_{3}$ & & & \\
\hline
\end{tabular}

Phase-1: Four-bar linkage $\mathrm{O}_{2} \mathrm{ABO}_{4}$

Fig.2. shows the first loop of the six bar mechanism. The link lengths are represented by the vectors for the ground link $\left(r_{1}\right)$, crank $\left(r_{2}\right)$, coupler $\left(r_{3}\right)$, and follower $\left(r_{4}\right)$. The range of angles for rotation of crank and follower is assumed.

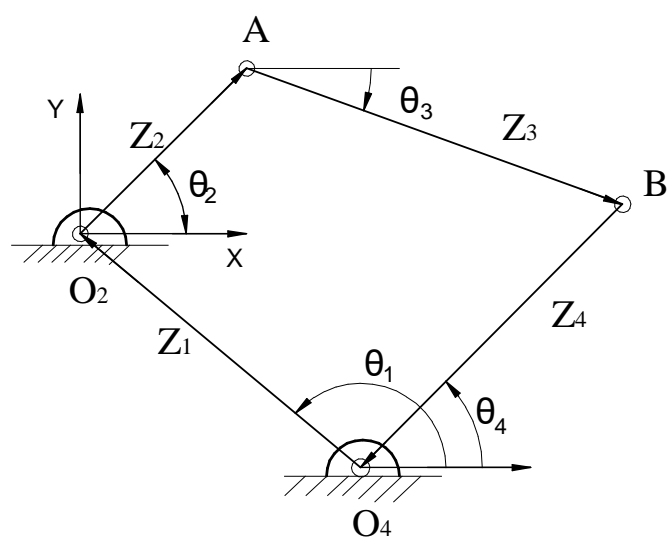

Fig.2. Parameters of a four bar mechanism

When three position synthesis is considered, Fruedenstein's equations for finding the lengths of the links of this four bar mechanism can be written as,

$$
\begin{aligned}
& Z_{4}=\frac{Z_{1}}{K_{1}}, \\
& Z_{2}=\frac{Z_{1}}{K_{2}} \text {, and } \\
& Z_{3}=\sqrt{2 K_{3} Z_{2} Z_{4}+Z_{1}^{2}+Z_{2}^{2}+Z_{4}^{2}} \\
& K_{3}=-\cos \left(\phi_{i}-\psi_{i}\right)-K_{1} \cos \phi_{i}-K_{2} \cos \psi_{i} \\
& K_{1}=\frac{\omega_{3} \omega_{5}-\omega_{2} \omega_{6}}{\omega_{1} \omega_{5}-\omega_{2} \omega_{4}} \text { and } K_{2}=\frac{\omega_{1} \omega_{6}-\omega_{3} \omega_{4}}{\omega_{1} \omega_{5}-\omega_{2} \omega_{4}} \text { where, } \\
& \quad \begin{array}{l}
\omega_{1}=\cos \phi_{1}-\cos \phi_{2}, \quad \omega_{4}=\cos \phi_{1}-\cos \phi_{3} \\
\omega_{2}=\cos \psi_{1}-\cos \psi_{2} \quad \omega_{5}=\cos \psi_{1}-\cos \psi_{3} \\
\quad \omega_{3}=-\cos \left(\phi_{1}-\psi_{1}\right)+\cos \left(\phi_{2}-\psi_{2}\right), \\
\quad \omega_{6}=-\cos \left(\phi_{1}-\psi_{1}\right)+\cos \left(\phi_{3}-\psi_{3}\right)
\end{array}
\end{aligned}
$$

The phase -2 of the six bar considered is a slider crank mechanism with the slider as the output link.For a slider crank mechanism, the Freudenstein's equations can be written as mentioned below. 


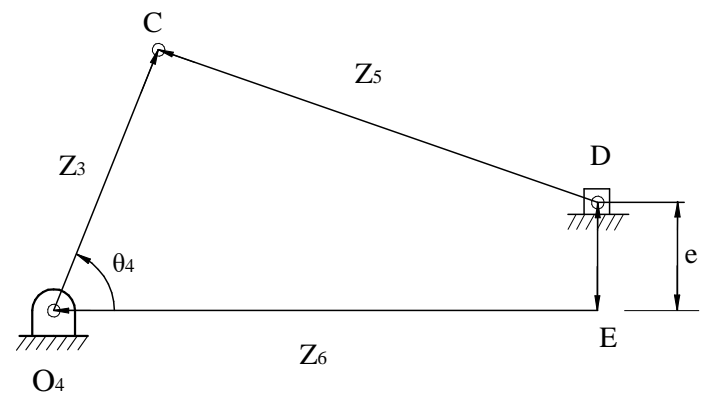

Fig 3. Parameters of a Slider Crank mechanism

In Fig. 3 an offset slider crank mechanism is shown having link vectors $Z_{3}, Z_{5}, Z_{6}$ and an eccentricity of e. The Freudenstein's equation for this linkage takes the form,

$K_{1} Z_{6} \cos \phi_{2}+K_{2} \sin \phi_{2}-K_{3}=Z_{6}^{2}, \quad$ where, $K_{1}=2 Z_{4}, K_{2}=2 Z_{4} e$ and $K_{3}=Z_{4}^{2}-Z_{5}^{2}+e^{2}$

The variation of the length of the coupler of the second phase slider crank mechanism is found by,

$\Delta b=-b+\sqrt{\left(s+a-2 a s \cos \theta_{2}\right)}$

\section{RESULTS AND DISCUSSION}

To understand the procedure adapted for the synthesis, let the function to be synthesized is $y=\sin (x)$, for which $0 \leq x \leq 90$ and $0 \leq y \leq 1$. The given values of the initial input and output angles and their ranges of operation are mentioned below in table-4.2.

Table -2 Values of different parameters for the phase -1

\begin{tabular}{|l|l|l|}
\hline Parameter & Notation & Value \\
\hline Initial angle of input link & $\varphi_{0}$ & $97^{0}$ \\
\hline Initial angle of output link & $\psi_{0}$ & $60^{0}$ \\
\hline Final angle of input link & $\varphi_{f}$ & $217^{0}$ \\
\hline Final angle of output link & $\psi_{f}$ & $120^{0}$ \\
\hline Range of input angle & $\Delta \varphi$ & $120^{0}$ \\
\hline Range of output angle & $\Delta \psi$ & $60^{\circ}$ \\
\hline
\end{tabular}

Using the procedure explained in the methodology section, the link lengths are calculated. The values of input and output angles calculated using Chebychev spacing are,

$\varphi_{1}=105^{0}, \varphi_{2}=157^{0}, \varphi_{3}=209^{0}$

$\psi_{1}=66.27^{0}, \psi_{2}=102.42^{0}, \psi_{3}=119.67^{0}$

The values of the link lengths calculated using Freudenstein's equations are, $r_{2}=29.0 \mathrm{~mm}, r_{3}=75.6 \mathrm{~mm}$ and $r_{4}=38.0 \mathrm{~mm}$.

To make this synthesized mechanism adjustable, one of the parameter is to be varied. As discussed earlier, the length of the coupler is taken as the variable. Then the value of the link length adjustment to be performed is then calculated using the equations developed in the methodology section. The computed values of the design variable $\Delta r_{3}$ against different values of the crank angles are given in table 4.2.3.

Table-3 Values of Design variable $\Delta r_{3}$ against the input crank angles

\begin{tabular}{|l|l|l|}
\hline $\begin{array}{l}\text { Input } \\
\text { angles }\left(\boldsymbol{\theta}_{\mathbf{2}}\right), \\
\text { degrees }\end{array}$ & $\begin{array}{l}\text { Variation of coupler } \\
\text { length }\left(\Delta \mathbf{r}_{\mathbf{3}}\right), \mathbf{m m}\end{array}$ & $\begin{array}{l}\text { Adjusted length } \\
\text { of coupler, r } \mathbf{3}, \\
\mathbf{m m}\end{array}$ \\
\hline 97 & -0.4525 & 75.1475 \\
\hline 105 & 0.0022 & 75.6022 \\
\hline 113 & 0.2792 & 75.8792 \\
\hline 121 & 0.4019 & 76.0019 \\
\hline 129 & 0.4104 & 76.0104 \\
\hline 137 & 0.3282 & 75.9282 \\
\hline 145 & 0.1986 & 75.7986 \\
\hline 153 & 0.0387 & 75.6387 \\
\hline 161 & -0.1221 & 75.4779 \\
\hline 169 & -0.2676 & 75.3324 \\
\hline 177 & -0.3675 & 75.2325 \\
\hline 185 & -0.4104 & 75.1896 \\
\hline 193 & -0.3828 & 75.2172 \\
\hline 201 & -0.2754 & 75.3246 \\
\hline
\end{tabular}

The values of $\Delta r_{3}$ given in the table-3 above indicate the amount of adjustment (increase / decrease) in the length of the coupler, $r_{3}$. The positive values indicate an increase in length to be made and the negative values suggest a decrease in length so that the function to be generated matches the actual function generated by the synthesized mechanism. Fig.4. shows the variation of the coupler length adjustment $\Delta r_{3}$ to be made for the corresponding input crank angle during the motion of the linkage. It indicates a need for the continuous adjustment so that linkage can follow the desired function. It can be seen that the highest value of adjustment is $0.5 \mathrm{~mm}$ on both positive and negative sides. 


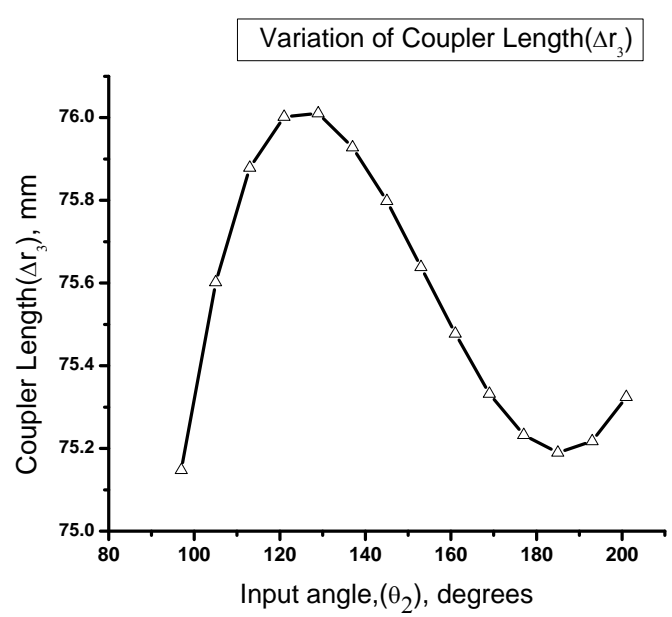

Fig. 4. Variation of coupler length adjustment $\Delta r_{3}$ against the input crank angle

As the phase -2 four bar is having the common link with the phase -1 it is necessary to know the output angles of the phase -1 which will be the input angles for the phase -2 . Table-4 gives the graphically measured corresponding values of the input and output angles for the first phase.

Table-4 Input and output angles for first phase of four bar mechanism

\begin{tabular}{|l|l|}
\hline $\begin{array}{l}\text { Input angles }\left(\boldsymbol{\theta}_{2}\right), \\
\text { degrees }\end{array}$ & $\begin{array}{l}\text { Output angles }\left(\boldsymbol{\theta}_{\mathbf{4}}\right), \\
\text { degrees }\end{array}$ \\
\hline 97 & 60.00 \\
\hline 105 & 66.27 \\
\hline 113 & 72.47 \\
\hline 121 & 78.54 \\
\hline 129 & 84.40 \\
\hline 137 & 90.00 \\
\hline 145 & 95.26 \\
\hline 153 & 100.14 \\
\hline 161 & 104.58 \\
\hline 169 & 108.54 \\
\hline 177 & 111.96 \\
\hline 185 & 114.81 \\
\hline 193 & 117.06 \\
\hline 201 & 118.69 \\
\hline
\end{tabular}

Path generated by the synthesized adjustable linkage

The path generated by the synthesized adjustable four bar linkage in the first phase is then found for different lengths of the adjustable parameter. The problem of synthesis for path generation usually requires computation of dimensions of link lengths such that a point on the coupler link of the mechanism traces out the desired trajectory. A path may have a prescribed shape, like circle, arc, elliptical, or a straight line. In the present work, the coupler curves generated for different lengths of the adjustable link are shown. Fig.5. shows the coupler path generated by the linkage without link length adjustment.

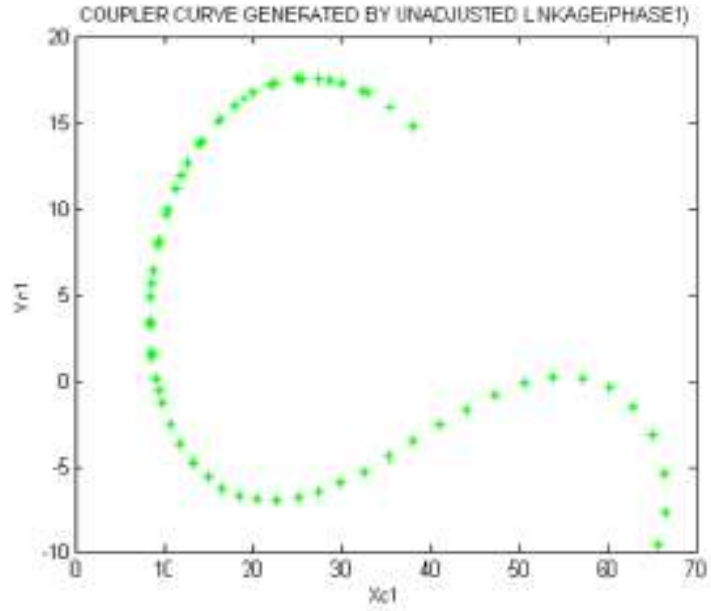

Fig.5. Coupler curve generated without link length adjustment

From the values of design variable $\Delta \mathrm{r}_{3}$ against the input crank angles given in table-3, it is found the maximum adjustment of the link length is 76.01 and it occurs at an input crank angle of $129^{\circ}$. The corresponding coupler curve is shown in Fig.6.

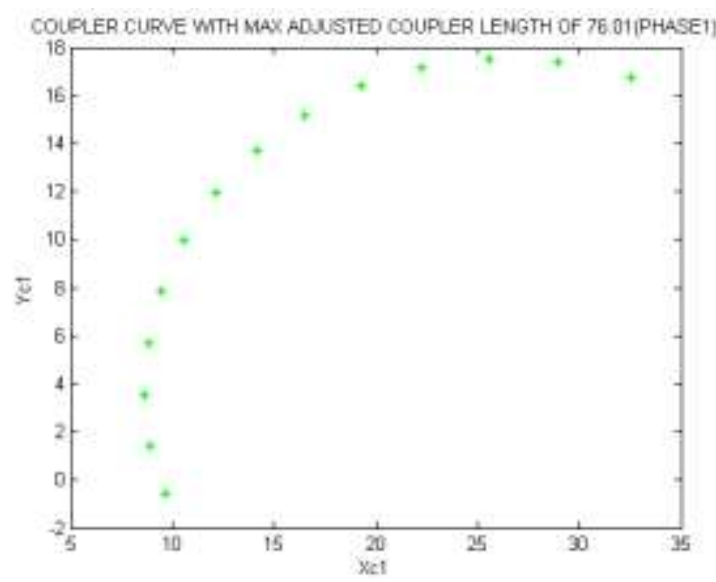

Fig.6. Coupler curve with a maximum adjusted coupler length

The path generated by the synthesized mechanism with the coupler adjusted to minimum length is given Fig.7. From table-3, it can be seen that the adjustment of the link length of the coupler on the negative side (shortening) is 75.14 and it occurs at an input crank angle of $97^{\circ}$.

Fig.8. represents the path generated by the linkage if the crank rotates by $360^{\circ}$. It can be observed here that the path of the coupler crosses over itself. 


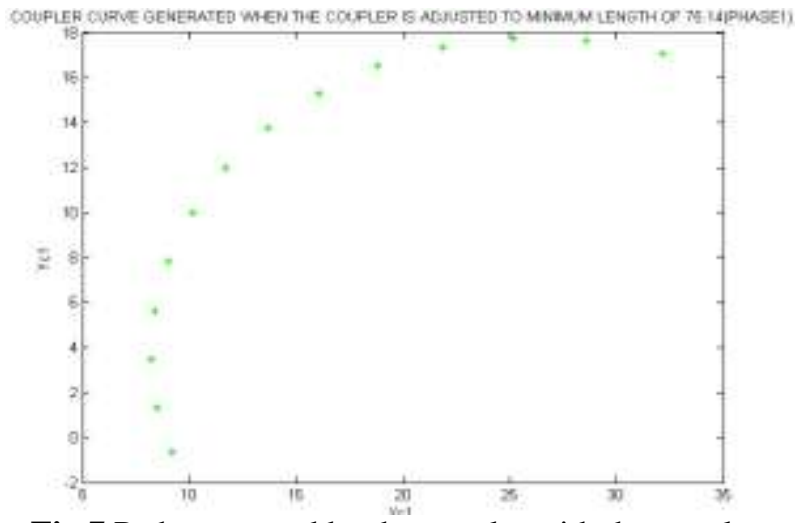

Fig.7.Path generated by the coupler with the coupler adjusted to minimum length

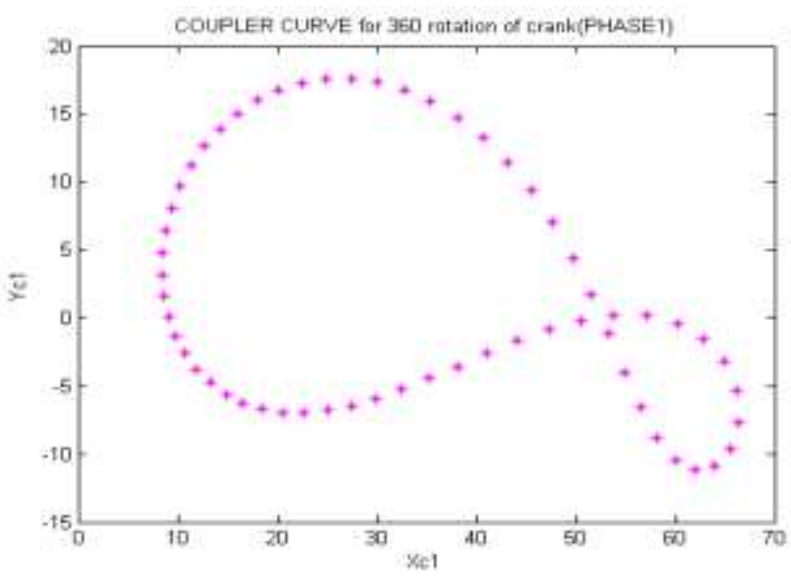

Fig.8.Path generated by the coupler when the crank rotates $360^{\circ}$

\section{Motion generated using the synthesized adjustable linkage}

Motion generation or rigid-body guidance requires that a moving body be guided through a series of prescribed positions. The body to be guided usually is a part of a coupler. A four-bar linkage can satisfy up to five prescribed positions for the motion generation problem. The adjustable four-bar linkage, on the other hand, can satisfy more than five given positions by making one or more parameters adjustable. The motion generated by the synthesized adjustable mechanism is shown below. When the coupler length is unadjusted the motion generated by the linkage is shown in fig 4.2.6. Figures 4.2.7 and 4.2.8 show the motion generated when the coupler is adjusted to a maximum adjusted length of $76.01 \mathrm{~mm}$ and a minimum adjusted length of $75.14 \mathrm{~mm}$.

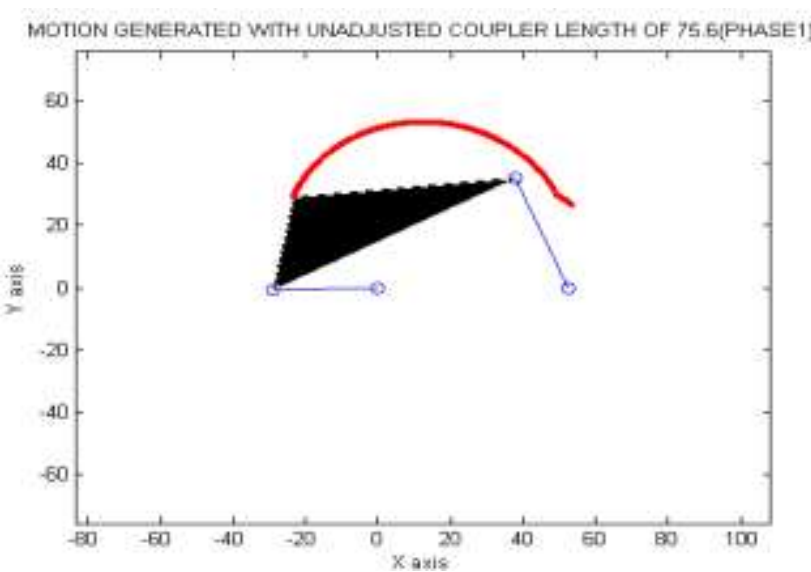

Fig.9. Motion generated with unadjusted length of the coupler

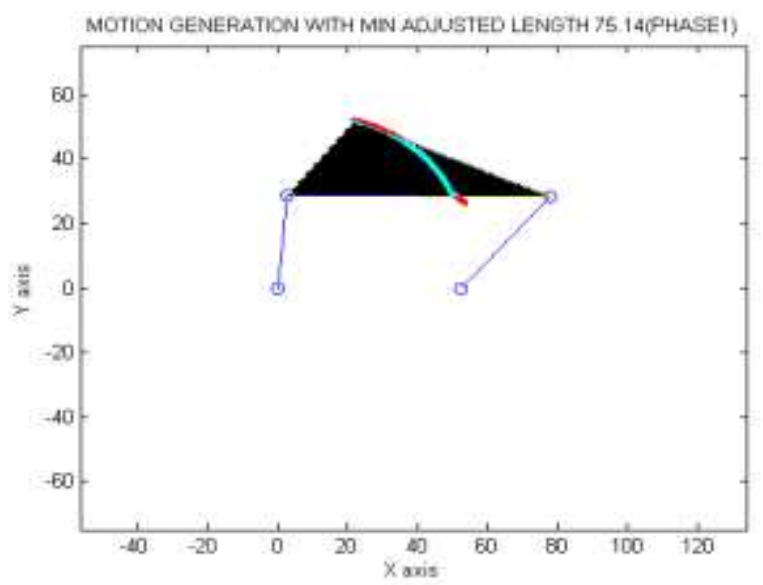

Fig.10. Motion generated with the minimum adjusted coupler length

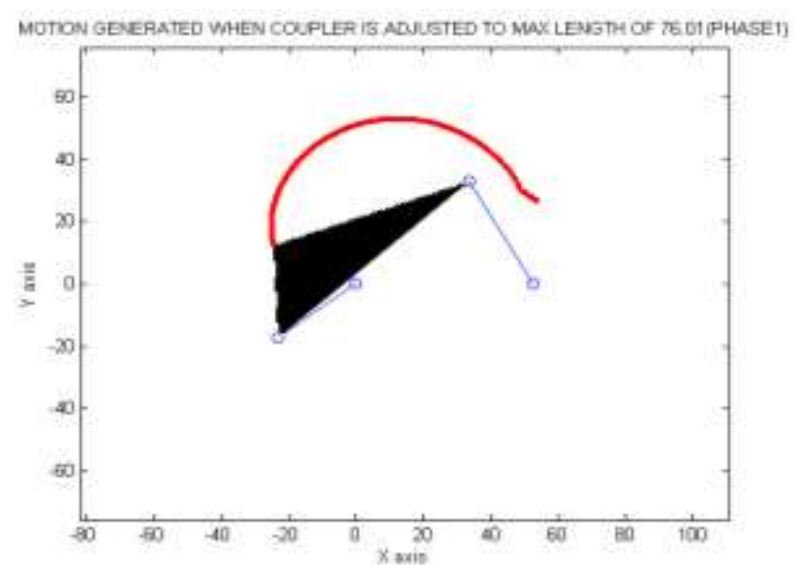

Fig.11.Motion generated with the maximum adjusted coupler length

\section{CONCLUSIONS}

In this paper, a methodology is presented for the Kinematic synthesis of planar four bar adjustable mechanisms using Chebychev's accuracy points and Fruedenstein's equations for three point function generation that satisfy a given input output relationship. The length of the coupler link is taken as the adjustable parameter. An analytical method of computing the value of this variable for a four bar crank 
rocker mechanism as well as a slider crank mechanism is presented. A numerical example explains the synthesis procedure developed in which a function to be generated is considered. The unknown link lengths of the mechanism are then found for given range of input and output link parameters and assumed values of link lengths. The value of the adjustable length of the coupler is then computed and the results are presented numerically as well as graphically. The method adopted is coded using MATLAB for quick computation and ease of use.

\section{REFERENCES}

[1]. Bonnell, R. D., and Cofer, J. A., (1966) Kinematic Synthesis of Adjustable Four-Bar Linkages, Journal of Applied Mechanics, Mar.

[2]. Naik D. P., Amarnath C., (1989) Adjustable four-bar function generators through five bar loop closure equations, Mechanism and Machine Theory 24 (6) $523-526$

[3]. Sandor G. N. and Erdman A. G., (1984), Advanced Mechanism Design: Analysis and Synthesis, Volume 2, Prentice Hall of India Private Ltd, New Delhi, EEE.

[4]. McGovern Joseph F. and Sandor George N., (1973), Kinematic Syndesis of Adjustable Mechanisms Part 1: Function Generation, Transactions of the ASME Journal of Engineering for Industry, PP 417-422,

[5]. Kota Sridhar, Soni A. H., Dev. P. Sathyadev, and Amir Shirkhodaie (contributors) and edited by Arthur Erdman, (1992), Modern Kinematics: Developments in the Last Forty Years, John Wiley \& Sons, Inc.

[6]. Chuenchom T., Kota S., (1997), Synthesis of Programmable Mechanisms using Adjustable Dyads, Journal of Mechanical Design, Vol. 119, pp232-237.

[7]. Shoup Terry E., (1984), The Design of an Adjustable Three Dimensional Slider Crank Mechanism, Mechanism and Machine Theory Vol. 19, No. 1, pp. 107-111. 